\title{
New mechanism for matter-antimatter asymmetry and connection with dark matter
}

\author{
Arnab Dasgupta $\odot,{ }^{1}$ P. S. Bhupal Dev ${ }^{2},{ }^{2}$ Sin Kyu Kang, ${ }^{1}$ and Yongchao Zhang $\odot^{2,3}$ \\ ${ }^{1}$ School of Liberal Arts, Seoul-Tech, Seoul 139-743, Korea \\ ${ }^{2}$ Department of Physics and McDonnell Center for the Space Sciences, Washington University, \\ St. Louis, Missouri 63130, USA \\ ${ }^{3}$ Department of Physics, Oklahoma State University, Stillwater, Oklahoma 74078, USA
}

(Received 17 December 2019; revised 16 July 2020; accepted 31 August 2020; published 14 September 2020)

\begin{abstract}
We propose a new mechanism for generating matter-antimatter asymmetry via the interference of treelevel diagrams only, where the imaginary part of the Breit-Wigner propagator for an unstable mediator plays a crucial role. We first derive a general result that a nonzero $C P$-asymmetry can be generated via at least two sets of interfering tree-level diagrams involving either $2 \rightarrow 2$ or $1 \rightarrow n$ (with $n \geq 3$ ) processes. We illustrate this point in a simple TeV-scale extension of the Standard Model with an inert Higgs doublet and right-handed neutrinos, along with an electroweak-triplet scalar field, where small Majorana neutrino masses are generated via a combination of radiative type-I and tree-level type-II seesaw mechanisms. The imaginary part needed for the required $C P$-asymmetry comes from the trilinear coupling of the inert doublet with the triplet scalar, along with the width of the triplet scalar mediator. The real part of the neutral component of the inert doublet serves as a cold dark matter candidate. The evolutions of the dark matter relic density and the baryon asymmetry are intimately related in this scenario.
\end{abstract}

DOI: 10.1103/PhysRevD.102.055009

\section{INTRODUCTION}

The observed asymmetry between the number densities of baryonic matter and antimatter in the universe [1] cannot be accounted for in the Standard Model (SM). Therefore, a viable baryogenesis mechanism is an essential ingredient for the success of any beyond SM scenario. The dynamical generation of baryon asymmetry requires three necessary (but not sufficient) Sakharov conditions [2] to be satisfied: (i) baryon number $(B)$ violation, (ii) $C$ and $C P$ violation, and (iii) out-of-equilibrium dynamics. A well-known mechanism that satisfies these conditions involves the $1 \rightarrow 2$ decays of a heavy particle, such as in grand unified theory (GUT) baryogenesis [3,4] or leptogenesis [5] (for reviews, see e.g., Refs. [6,7]). To obtain a baryon/lepton asymmetry in these $1 \rightarrow 2$ decay scenarios, one must consider the interference between tree- and loop-level diagrams. Furthermore, some particles in the loop must be able to go on-shell, and the interaction between the intermediate on-shell particles and the final-state particles should correspond to a net change in baryon/lepton number for the net asymmetry to be nonzero; this is known as the

Published by the American Physical Society under the terms of the Creative Commons Attribution 4.0 International license. Further distribution of this work must maintain attribution to the author(s) and the published article's title, journal citation, and DOI. Funded by SCOAP ${ }^{3}$.
Nanopoulos-Weinberg theorem [8] (see also Refs. [9,10]). Similar interference effects between tree and loop-level diagrams have also been considered for generating the baryon asymmetry from $2 \rightarrow 2$ scattering [11-14] or annihilation [15-20] processes.

In this paper, we argue that the interference between tree and loop-level diagrams is not the only way to generate a nonzero asymmetry from out-of-equilibrium heavy particle decays/annihilations. We propose a new mechanism where it suffices to consider two sets of interfering diagrams at the tree-level only. This can be achieved through tree-level $2 \rightarrow 2$ scattering or $1 \rightarrow n$ (with $n \geq 3$ ) decay processes mediated by unstable particles. Then the $C P$-asymmetry can be generated from the complex couplings and the propagator widths [see Eq. (3) below], which could even be resonantly enhanced when the center-of-mass energy is close to the propagator mass.

To illustrate our new mechanism, we will consider a simple realistic model at $\mathrm{TeV}$-scale, namely, combining the scotogenic model [21] (with an inert Higgs doublet and right-handed neutrinos) and the type-II seesaw framework [22-26] (with an $S U(2)_{L}$-triplet scalar) for small Majorana neutrino masses. For our parameter choice of the model, the $C P$-asymmetry originates from the complex trilinear coupling of the inert Higgs doublet with the triplet scalar, along with the imaginary part of the triplet scalar mediator width. Stabilized by a discrete $Z_{2}$ symmetry, the neutral component of the inert doublet scalar plays the role of a TeV-scale 
weakly interacting dark matter (DM) candidate. Adopting three benchmark points (BPs), we illustrate that the generation of baryon asymmetry via leptogenesis in this model is intimately correlated with the DM relic density. It is also found that successful leptogenesis sets limits on the triplet vacuum expectation value (VEV) $v_{\Delta}$ and the trilinear scalar coupling $\left|\mu_{\eta \Delta}\right|$ (see Fig. 7)—a feature that could be directly tested at future high-energy colliders.

The rest of this paper is organized as follows. Our general mechanism of attaining the $C P$ asymmetry without having explicit loop diagrams is explained in Sec. II. The scotogenic plus type-II seesaw model is introduced in Sec. III. The lepton asymmetry generation in this model is detailed in Sec. IV. The collider signatures are touched upon in Sec. V. Our conclusions are given in Sec. VI. The scalar potential and scalar masses are collected in the Appendix A. The relevant thermal cross sections used in our analysis are given in Appendix B. The thermal cross section relevant for the asymmetry in the narrow-width approximation is given in Appendix C.

\section{THE GENERAL MECHANISM}

We propose that a net lepton or baryon asymmetry can be generated from the interference effect of two sets of treelevel decay or scattering diagrams with the same initial and final states, as long as the following two conditions are satisfied:

(i) There is a net nonzero lepton or baryon number between the initial and final states. ${ }^{1}$

(ii) At least one set of decay or scattering amplitudes is complex such that the squared amplitudes for particles and antiparticles are different, giving rise to a net $C P$-asymmetry.

The simplest way to achieve this is through $2 \rightarrow 2$ scatterings (see Fig. 1) or $1 \rightarrow 3$ decays (see Fig. 2) involving two different intermediate state particles, with the outgoing particles (or decay products) carrying a net nonzero baryon or lepton number. Without loss of generality, we focus here on the simplest $2 \rightarrow 2$ scattering case with the initial states $i_{1}, i_{2}$ and with only two subprocesses for the final states $f_{1}, f_{2}$ (here $i_{1,2}$ and $f_{1,2}$ generically stand for bosons and/or fermions), mediated by intermediatestate particles of mass $m_{1}$ and $m_{2}$, respectively. The total amplitude for the process $i_{1} i_{2} \rightarrow f_{1} f_{2}$ can be written as

$$
\mathcal{M}=\left(\mathcal{C}_{1} \mathcal{M}_{1}+\mathcal{C}_{2} \mathcal{M}_{2}\right) \mathcal{W}
$$

where $\mathcal{C}_{i}$ contain only the couplings, $\mathcal{W}$ contains the wave functions for the incoming and outgoing particles and $\mathcal{M}_{i}$

\footnotetext{
${ }^{1}$ In principle, this condition can be somewhat relaxed if we consider flavor-dependent asymmetries, with zero net lepton or baryon number in the final state, as in flavored leptogenesis (for recent reviews, see e.g., Refs. [27,28]). For simplicity, here we will not consider such flavor-dependent effects.
}

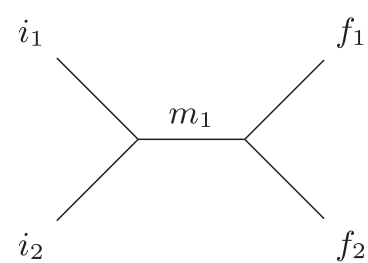

(a)

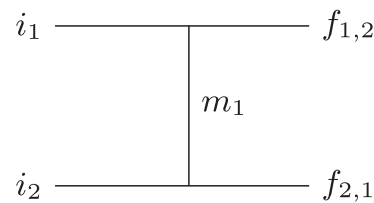

(c)

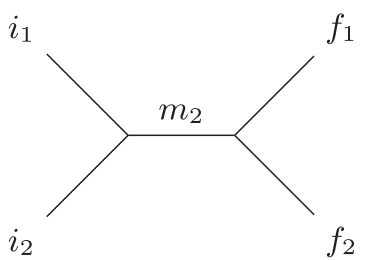

(b)

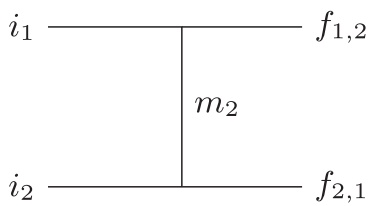

(d)
FIG. 1. Generic topologies for tree-level $2 \rightarrow 2$ subprocesses that can give rise to a nonzero lepton or baryon asymmetry. Here $i_{1,2}$ and $f_{1,2}$ are respectively the initial and final states, and $m_{1,2}$ are the masses of two different mediators.

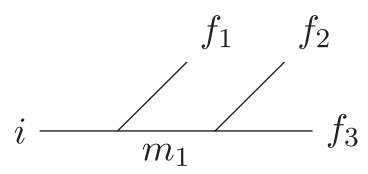

(a)

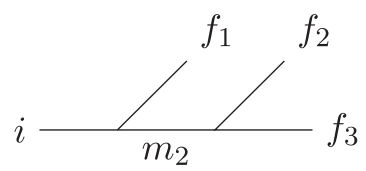

(b)
FIG. 2. Generic topologies for tree-level $1 \rightarrow 3$ subprocesses that can give rise to a nonzero lepton or baryon asymmetry. Here $i$ and $f_{1,2,3}$ are respectively the initial and final states, and $m_{1,2}$ are the masses of two different mediators.

stand for the rest of the subamplitudes. The corresponding amplitude for the conjugate process $\bar{i}_{1} \bar{i}_{2} \rightarrow \bar{f}_{1} \bar{f}_{2}$ is ${ }^{2}$

$$
\overline{\mathcal{M}}=\left(\mathcal{C}_{1}^{*} \mathcal{M}_{1}+\mathcal{C}_{2}^{*} \mathcal{M}_{2}\right) \mathcal{W}^{*}
$$

Comparing the modular squares of the amplitudes, we obtain the $C P$-asymmetry factor

$$
\begin{aligned}
\delta & \equiv|\mathcal{M}|^{2}-|\overline{\mathcal{M}}|^{2} \\
& =-4 \operatorname{Im}\left[\mathcal{C}_{1} \mathcal{C}_{2}^{*}\right] \operatorname{Im}\left[\mathcal{M}_{1} \mathcal{M}_{2}^{*}\right]|\mathcal{W}|^{2},
\end{aligned}
$$

where $\operatorname{Im}\left[\mathcal{C}_{1} \mathcal{C}_{2}^{*}\right]$ is the imaginary part coming from the couplings, which is required to be nonzero for $C P$ violation, and $\operatorname{Im}\left[\mathcal{M}_{1} \mathcal{M}_{2}^{*}\right]$ incorporates the imaginary part from the subamplitudes $\mathcal{M}_{1,2}$, which is reminiscent of the imaginary part coming from the interference of tree and loop-level diagrams in the $1 \rightarrow 2$ decay scenario. Equation (3) is a general result applicable to $2 \rightarrow 2$ scatterings, as well as $1 \rightarrow n$ decays (for $n \geq 3$ ).

\footnotetext{
${ }^{2}$ Note that the $C P T$ theorem only guarantees the equivalence of rates for $i_{1} i_{2} \rightarrow f_{1} f_{2}$ and $\bar{f}_{1} \bar{f}_{2} \rightarrow \bar{i}_{1} \bar{i}_{2}$.
} 
In the tree-level $2 \rightarrow 2$ processes shown in Fig. 1, we have only one source for the complex subamplitudes, namely, the finite widths of the mediators. One may argue that the finite width is also a loop-induced effect for unstable mediators, since it is related to the imaginary part of self-energy $[29,30]$. However, the crux of our new mechanism is that we only require a nonzero width, whereas the $1 \rightarrow 2$ decay case needs both nonzero width and interference between tree and loop (self-energy and/or vertex correction) diagrams. In general, the subamplitudes $\mathcal{M}_{1,2}$ for the processes in Fig. 1 can be written as

$$
\mathcal{M}_{j}=\frac{A_{j}}{x_{j}-m_{j}^{2}+i m_{j} \Gamma_{j}},
$$

with $j=1,2, x_{j}=s, t, u$ the Mandelstam variables, $m_{j}$ and $\Gamma_{j}$ respectively the mediator masses and widths, and $A_{j}$ some arbitrary real parameters. The imaginary component of the product of subamplitudes appearing in Eq. (3) can then be written as

$\operatorname{Im}\left[\mathcal{M}_{1} \mathcal{M}_{2}^{*}\right]=\frac{A_{1} A_{2}\left[\left(x_{1}-m_{1}^{2}\right) m_{2} \Gamma_{2}-\left(x_{2}-m_{2}^{2}\right) m_{1} \Gamma_{1}\right]}{\left[\left(x_{1}-m_{1}^{2}\right)^{2}+m_{1}^{2} \Gamma_{1}^{2}\right]\left[\left(x_{2}-m_{2}^{2}\right)^{2}+m_{2}^{2} \Gamma_{2}^{2}\right]}$,

which is nonvanishing as long as $\left(x_{1}-m_{1}^{2}\right) m_{2} \Gamma_{2} \neq\left(x_{2}-\right.$ $\left.m_{2}^{2}\right) m_{1} \Gamma_{1}$ in the numerator. With the imaginary part of the couplings $\operatorname{Im}\left[\mathcal{C}_{1} \mathcal{C}_{2}^{*}\right] \neq 0$, we can then produce a nonzero asymmetry [cf. Eq. (3)]. This general argument holds, irrespective of the specific subprocesses or the model details.

For the tree-level $2 \rightarrow 2$ case in Fig. 1, we can have three distinct possibilities for the two subprocesses to realize $\operatorname{Im}\left[\mathcal{M}_{1} \mathcal{M}_{2}^{*}\right] \neq 0$ in Eq. (5):

(i) If both subprocesses are in the $s$-channel [cf. Fig. 1 (a)+(b)], one just needs to replace $x_{1,2}$ by $s$ in Eq. (5). In this case, the $C P$-asymmetry factor $\delta$ in Eq. (3) can be largely enhanced in the vicinity of resonance (s), with $s-m_{i}^{2} \simeq m_{i} \Gamma_{i}$ (with $i=1,2$ ), similar to the enhancement effect in resonant leptogenesis [31,32].

(ii) If one of the subamplitudes is in the $s$-channel and the other one in the $t$ - or $u$-channel [cf. Fig. 1(a)+(d) or (b)+(c)], one can safely neglect the imaginary part for the $t$ - or $u$-channel propagator. For concreteness, we take $\mathcal{M}_{1}$ as the $s$-channel and $\mathcal{M}_{2}$ as the $x$-channel $(x=t$ or $u)$ amplitude. In this case, Eq. (5) can be simplified to

$$
\operatorname{Im}\left[\mathcal{M}_{1} \mathcal{M}_{2}^{*}\right] \simeq-\frac{A_{1} A_{2} m_{1} \Gamma_{1}}{\left[\left(s-m_{1}^{2}\right)^{2}+m_{1}^{2} \Gamma_{1}^{2}\right]\left(x-m_{2}^{2}\right)},
$$

which is proportional to the $s$-channel mediator width $\Gamma_{1}$. Here also the $C P$-asymmetry could be largely enhanced at the $s$-channel resonance, i.e., $s-m_{1}^{2} \simeq m_{1} \Gamma_{1}$.

(iii) If both subprocesses are in the $t$ - or $u$-channel [cf. Fig. 1(c)+(d)], then the width terms in the denominator of Eq. (5) can be neglected, i.e.,

$$
\begin{aligned}
& \operatorname{Im}\left[\mathcal{M}_{1} \mathcal{M}_{2}^{*}\right] \\
& \quad \simeq \frac{A_{1} A_{2}\left[\left(x_{1}-m_{1}^{2}\right) m_{2} \Gamma_{2}-\left(x_{2}-m_{2}^{2}\right) m_{1} \Gamma_{1}\right]}{\left(x_{1}-m_{1}^{2}\right)^{2}\left(x_{2}-m_{2}^{2}\right)^{2}} .
\end{aligned}
$$

In this case, the $C P$-asymmetry is suppressed by the ratio $m_{i} \Gamma_{i} /\left(x_{j}-m_{j}^{2}\right)$ with $i, j=1,2$.

In the next section, we will consider an explicit example that realizes the possibility (ii) discussed above.

\section{AN EXAMPLE}

To illustrate our new mechanism in a minimal realistic extension of SM, we consider an amalgamation of the scotogenic model [21] and type-II seesaw [22-26] mechanisms at TeV-scale. For the purpose of scotogenic mechanism, an inert $S U(2)_{L}$-doublet scalar $\eta \equiv\left(\eta^{+}, \eta^{0}\right)$ and three right-handed neutrinos (RHNs) $N_{i}$ (with $i=1,2,3$ ) are introduced. To implement type-II seesaw, an $S U(2)_{L}$-triplet scalar $\Delta \equiv\left(\Delta^{++}, \Delta^{+}, \Delta^{0}\right)$ is added. The inert doublet $\eta$ and the three RHNs $N_{i}$ are odd under a discrete $Z_{2}$ symmetry, while all other particles are even. In this model, we assume the RHNs, as well as the triplet scalar components, are heavier than the $\eta$ scalars, so any asymmetry generated by the conventional decays of $N$ and/or $\Delta$ is not relevant at the temperature scale of interest. An added advantage of our mechanism is that the lightest neutral component $\eta^{0}$ plays the role of DM [21], with its relic density intimately connected to the lepton asymmetry. A nonminimal coupling of the inert doublet to gravity can also successfully accommodate inflation [33,34].

The relevant Yukawa couplings are given by the Lagrangian

$$
-\mathcal{L}_{Y}=Y_{i \alpha}^{N} \tilde{\eta}^{\dagger} L_{\alpha} N_{i}+Y_{\alpha \beta}^{\Delta} \overline{L_{\alpha}^{C}} \Delta L_{\beta}+\text { H.c. },
$$

with $L \equiv(\nu, \ell)$ being the SM lepton doublet, $C$ the charge conjugation operator, $\tilde{\eta}=i \sigma_{2} \eta^{*}$ ( $\sigma_{2}$ being the second Pauli matrix), $\alpha, \beta=e, \mu, \tau$ the lepton flavor indices, and $i=1$, 2,3 the RHN mass indices. For simplicity, we assume there is no mixing nor $C P$ violation in the RHN sector. The mass parameter $\mu_{\eta \Delta}$ in the scalar potential

$$
V \supset \mu_{\eta \Delta} \eta^{\dagger} \Delta^{\dagger} \tilde{\eta}+\text { H.c. }
$$

is chosen to be complex, which is crucial for the $C P$ asymmetry [cf. Eq. (3)]. The full scalar potential and the resultant physical scalar masses are collected in Appendix A.

In this setup, the neutrino mass is generated from both loop-level scotogenic and tree-level type-II seesaw 
mechanisms, which are induced respectively by the Yukawa couplings $Y^{N}$ and $Y^{\Delta}$ given in Eq. (8):

$$
m_{\nu}=\left(Y^{N}\right)^{\top} \Lambda Y^{N}+Y^{\Delta} v_{\Delta},
$$

where $\Lambda$ is an effective loop-suppressed RHN mass scale, given by $[21,35]$

$$
\begin{aligned}
\Lambda_{i i}= & \frac{m_{N_{i}}}{16 \pi^{2}}\left[\frac{m_{\eta_{R}}^{2}}{m_{N_{i}}^{2}-m_{\eta_{R}}^{2}} \ln \left(\frac{m_{N_{i}}^{2}}{m_{\eta_{R}}^{2}}\right)\right. \\
& \left.-\frac{m_{\eta_{\mathrm{I}}}^{2}}{m_{N_{i}}^{2}-m_{\eta_{I}}^{2}} \ln \left(\frac{m_{N_{i}}^{2}}{m_{\eta_{I}}^{2}}\right)\right] .
\end{aligned}
$$

We have assumed that the RHNs do not mix with each other, therefore $\Lambda$ is a diagonal matrix. The Yukawa couplings in Eq. (10) are related to the neutrino oscillation data, $\Lambda$ and the triplet $\operatorname{VEV}\left\langle\Delta^{0}\right\rangle=v_{\Delta}$ as follows:

$$
\begin{aligned}
& Y_{i \alpha}^{N}=F_{\mathrm{I}}^{1 / 2}\left(\Lambda^{-1 / 2} \mathcal{O} \hat{m}_{\nu}^{1 / 2} U_{\mathrm{PMNS}}^{\dagger}\right)_{i \alpha}, \\
& Y_{\alpha \beta}^{\Delta}=F_{\mathrm{II}} v_{\Delta}^{-1}\left(U_{\mathrm{PMNS}}^{*} \hat{m}_{\nu} U_{\mathrm{PMNS}}^{\dagger}\right)_{\alpha \beta},
\end{aligned}
$$

where $\hat{m}_{\nu}=\left\{m_{\nu_{1}}, m_{\nu_{2}}, m_{\nu_{3}}\right\}$ the diagonal neutrino mass eigenvalues, and $U_{\mathrm{PMNS}}$ the PMNS lepton mixing matrix. In Eq. (12) we have used the Casas-Ibarra parametrization [36] for the coupling $Y^{N}$, with $\mathcal{O}$ an arbitrary orthogonal matrix. $F_{\mathrm{I}}$ and $F_{\mathrm{II}}$ are the fractions of contributions to neutrino mass matrix from the radiative scotogenic and tree-level type-II seesaw mechanisms respectively, with $F_{\text {I }}+F_{\text {II }}=1$.

\section{GENERATION OF LEPTON ASYMMETRY AND DARK MATTER RELIC DENSITY}

As stated above, the matter asymmetry is generated from the interference effect between two tree-level diagrams, which are shown in Fig. 3 for our scotogenic type-II seesaw model with $m_{\Delta} \gtrsim 2 m_{\eta}$. In particular, we analyze the $2 \rightarrow 2$ $\Delta L=2$ scattering processes

$$
\eta \eta \rightarrow L_{\alpha} L_{\beta}
$$

which include $\quad \eta^{ \pm} \eta^{ \pm} \rightarrow \ell_{\alpha}^{ \pm} \ell_{\beta}^{ \pm}, \quad \eta^{0} \eta^{ \pm} \rightarrow \ell_{\alpha}^{ \pm} \nu_{\beta} \quad$ and $\eta^{0} \eta^{0} \rightarrow \nu_{\alpha} \nu_{\beta}$. These processes can be mediated by an $s$ channel triplet scalar $\Delta$, and also by RHNs $N_{i}$ in the $t$ - and $u$-channels, as shown in Fig. 3. The effective $C P$-asymmetry factor [cf. Eq. (6)] is given by

\footnotetext{
${ }^{3}$ In the opposite regime where $m_{\Delta}<2 m_{\eta}$, an asymmetry can be generated via the interference between tree-level $\Delta \rightarrow L L$ decay mediated by the Yukawa coupling $Y^{\Delta}$ and the vertex correction to this decay induced by two $\eta$ 's and a $N$ mediated by the couplings $\mu_{\eta \Delta}$ and $Y^{N}$ respectively.
}

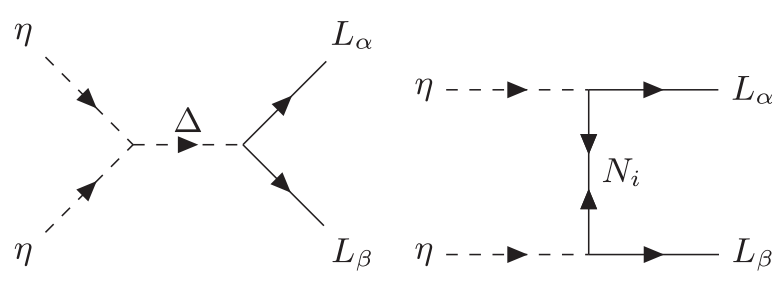

FIG. 3. Feynman diagrams for the $2 \rightarrow 2$ scattering processes $\eta \eta \rightarrow L_{\alpha} L_{\beta}$ in our example model.

$$
\begin{aligned}
\delta= & 4 \sum_{i} \operatorname{Im}\left[\mu_{\eta \Delta}\left\{Y^{N} Y^{\Delta^{*}}\left(Y^{N}\right)^{\top}\right\}_{i i}\right] \\
& \times \frac{s m_{N_{i}} m_{\Delta} \Gamma_{\Delta}}{\left(s-m_{\Delta}^{2}\right)^{2}+m_{\Delta}^{2} \Gamma_{\Delta}^{2}}\left[\frac{1}{t-m_{N_{i}}^{2}}+\frac{1}{u-m_{N_{i}}^{2}}\right],
\end{aligned}
$$

where $\Gamma_{\Delta}$ is the triplet scalar width. With the width in the numerator of Eq. (15), the asymmetry in this simple model can be viewed as the interference effect of RHN-mediated tree-level diagram in the right panel of Fig. 3 and the oneloop correction to the triplet propagator in the $s$-channel, where the $s$-channel process corresponds to the following subprocesses

$$
\eta \eta \rightarrow \Delta^{(*)}, \quad \Delta^{(*)} \rightarrow L L
$$

which incorporate the conventional one-loop decay of (onshell) triplet scalars into a lepton pair.

From an effective field theory (EFT) perspective, if we integrate out the heavy mediator masses $m_{\Delta}$ and $m_{N_{i}}$, the width effect has to be consistently incorporated into the effective coupling. This can be understood by inserting a self-energy diagram in the $\Delta$-propagator in Fig. 3 and then integrating out the resulting two $\Delta$ propagators to obtain a loop-level effective coupling that includes the width of $\Delta$, giving rise to a non-vanishing asymmetry.

In Eq. (15), the imaginary part of the product of the Yukawa couplings $Y^{N}, Y^{\Delta}$ [cf. Eq. (8)] and the trilinear coupling $\mu_{\eta \Delta}$ [cf. Eq. (9)] can be parametrized using Eqs. (12) and (13) as follows:

$$
\begin{aligned}
& \operatorname{Im}\left[\mu_{\eta \Delta}\left\{Y^{N} Y^{\Delta^{*}}\left(Y^{N}\right)^{\top}\right\}_{i i}\right] \\
& \quad=F_{\mathrm{I}} F_{\mathrm{II}} v_{\Delta}^{-1} \operatorname{Im}\left[\mu_{\eta \Delta}\left\{\Lambda^{-1 / 2} \mathcal{O} \hat{m}_{\nu}^{2} \mathcal{O}^{\top} \Lambda^{-1 / 2}\right\}_{i i}\right]
\end{aligned}
$$

In general, the orthogonal $\mathcal{O}$-matrix might be complex, thus potentially contributing to the imaginary part in Eq. (17).

It is interesting that part of the same $2 \rightarrow 2$ processes in Eq. (14) containing $\eta^{0}$ contributes also to the (co)annihilation of DM particles. In this sense, the time evolutions of DM relic density and the lepton asymmetry are correlated, as we will see below. The freeze-out mechanism for DM is identical to the standard inert doublet case $[37,38]$, where DM annihilates into the SM particles. 


\section{A. Boltzmann equations}

The cogenesis of DM relic density and leptonic asymmetry is governed by the coupled Boltzmann equations

$$
\begin{aligned}
\frac{\mathrm{d} Y_{\eta}}{\mathrm{d} z}= & \frac{-s}{H(z) z}\left[\left(Y_{\eta}^{2}-\left(Y_{\eta}^{\mathrm{eq}}\right)^{2}\right)\langle\sigma v\rangle(\eta \eta \rightarrow \mathrm{SMSM})\right], \\
\frac{\mathrm{d} Y_{\Delta L}}{\mathrm{~d} z}= & \frac{s}{H(z) z}\left[\left(Y_{\eta}^{2}-\left(Y_{\eta}^{\mathrm{eq}}\right)^{2}\right)\langle\sigma v\rangle_{\delta}(\eta \eta \rightarrow L L)\right. \\
& -2 Y_{\Delta L} Y_{\ell}^{\mathrm{eq}} r_{\eta}^{2}\langle\sigma v\rangle_{\mathrm{tot}}(\eta \eta \rightarrow L L) \\
& \left.-2 Y_{\Delta L} Y_{\eta}^{\mathrm{eq}}\langle\sigma v\rangle(\eta \bar{L} \rightarrow \eta L)\right],
\end{aligned}
$$

where $z=m_{\eta} / T, Y_{i}^{(\mathrm{eq})} \equiv n_{i}^{(\mathrm{eq})} / s$ are the normalized number densities (in equilibrium) for the particles $i$ ( $s$ being the entropy density), $Y_{\Delta L}=Y_{L}-Y_{\bar{L}}, r_{\eta}=Y_{\eta}^{\mathrm{eq}} / Y_{\ell}^{\mathrm{eq}}$, and

$$
H(z)=\sqrt{\frac{8 \pi^{3} g_{*}}{90}} \frac{m_{\eta}^{2}}{z^{2} M_{\mathrm{Pl}}}
$$

with $M_{\mathrm{Pl}}$ the Planck scale and $g_{*}$ the number of relativistic degrees of freedom at temperature $T$. Here the $\langle\sigma v\rangle$ 's are the thermally averaged annihilation/scattering rates: $\langle\sigma v\rangle(\eta \eta \rightarrow \mathrm{SMSM})$ is the $\mathrm{DM}$ annihilation rate, and $\langle\sigma v\rangle_{\mathrm{tot}, \delta}(\eta \eta \rightarrow L L)$ are respectively given by

$$
\begin{aligned}
\langle\sigma v\rangle_{\mathrm{tot}}(\eta \eta \rightarrow L L) \equiv & \langle\sigma v\rangle(\eta \eta \rightarrow L L) \\
& +\langle\sigma v\rangle\left(\eta^{*} \eta^{*} \rightarrow \bar{L} \bar{L}\right), \\
\langle\sigma v\rangle_{\delta}(\eta \eta \rightarrow L L) \equiv & \langle\sigma v\rangle(\eta \eta \rightarrow L L) \\
& -\langle\sigma v\rangle\left(\eta^{*} \eta^{*} \rightarrow \bar{L} \bar{L}\right) .
\end{aligned}
$$

The expressions for all the thermal cross sections appearing in Eqs. (18) and (19) are collected in Appendices B and C.

Evaluating the Boltzmann equations above, one can obtain the lepton asymmetry $Y_{\Delta L}(z)$, which is then converted to baryon asymmetry $Y_{\Delta B}=-(28 / 51) Y_{\Delta L}$ [39] via the standard electroweak sphaleron processes [40] at the sphaleron transition temperature $T_{\mathrm{sph}}=(131.7 \pm 2.3) \mathrm{GeV}$ [41]. In an analogous way, one can also calculate the evolution of the DM density $Y_{\eta}$ from Eq. (18) and get the final relic abundance $\Omega_{\mathrm{DM}} h^{2}=2.755 \times 10^{8} Y_{\eta}\left(m_{\eta} / \mathrm{GeV}\right)$ at DM freeze-out temperature $T_{f} \simeq m_{\eta} / 20$.

We note here that our mechanism for generating the lepton asymmetry and DM relic density simultaneously is similar to the WIMPy baryogenesis mechanism [17]. A crucial criterion for achieving successful asymmetry in both cases is that the washout of the asymmetry processes must freeze-out before the freeze-out of the DM annihilation processes, i.e., $\langle\sigma v\rangle_{\text {tot }}(\eta \eta \rightarrow L L)<\langle\sigma v\rangle(\eta \eta \rightarrow$ SMSM $)$. In Ref. [17], both washout and DM freeze-out are governed by the same final states; therefore, one of the final states is required to be massive to satisfy the above freeze-out condition. In our case, however, the dominant process for DM freeze-out is $\eta \eta \rightarrow$ $W^{+} W^{-}$via $S U(2)_{L}$ gauge interaction, whereas the dominant washout process is $\eta \eta \rightarrow L L$ via the Yukawa couplings; therefore, we can satisfy the freeze-out condition for suitable choice of the Yukawa couplings without requiring any of the final states to be massive.

\section{B. Numerical results}

The three BPs used in our numerical analysis of the baryon asymmetry $Y_{\Delta B}$ and DM relic density $\Omega_{\mathrm{DM}} h^{2}$ [cf. Fig. 5] are collected in Table I. These are obtained by implementing our model in SARAH 4 [42] and after checking consistency with all lepton flavor violating constraints using SPheno 4.0.4 [43]. The observed value of $\mathrm{DM}$ relic density is obtained in each case by fixing the Higgs-DM quartic couplings $\lambda_{H \eta}=-\lambda_{H \eta}^{\prime}$ in Eq. (A1) for a given mass scale $\mu_{\eta}$ as shown in Table I. This assumption is taken in order to ensure the mass of the charged scalar is always higher than the neutral scalar masses (i.e., $\eta_{R}$ and $\eta_{I}$ ). All the quartic couplings in Eq. (A1) not listed in this table are set to be zero.

We solve the Boltzmann equations (18) and (19) numerically for the three representative BPs in Table I. We assume $F_{\mathrm{I}}=F_{\text {II }}=1 / 2$ in Eqs. (12) and (13), i.e., equal contributions from scotogenic and type-II seesaw to neutrino masses. This choice maximizes the $C P$-asymmetry in Eq. (17), subject to keeping other factors the same. In addition, the $\mathcal{O}$ matrix is taken to be identity, so that $\mathcal{O} \hat{m}_{\nu}^{2} \mathcal{O}^{\top}=\hat{m}_{\nu}^{2}$, and the mass parameter $\mu_{\eta \Delta}$ is assumed to be purely imaginary in Eq. (17). In doing so, the contribution for the asymmetry

TABLE I. Three BPs for the numerical analysis. All the quartic couplings in Eq. (A1) not listed in this table are set to be zero. Here $\Delta m_{\eta^{0}}=m_{\eta_{R}}-m_{\eta_{I}}$ is the mass splitting between the two scalars $\eta_{R}$ and $\eta_{I}$.

\begin{tabular}{lccc}
\hline \hline & $\mathrm{BP} 1$ & $\mathrm{BP} 2$ & $\mathrm{BP} 3$ \\
\hline$v_{\Delta}$ & $1 \mathrm{keV}$ & $1 \mathrm{keV}$ & $1 \mathrm{keV}$ \\
$\mu_{\eta}$ & $600 \mathrm{GeV}$ & $1 \mathrm{TeV}$ & $1.5 \mathrm{TeV}$ \\
$\mu_{H \Delta}$ & $33.6 \mathrm{keV}$ & $93.5 \mathrm{keV}$ & $210 \mathrm{keV}$ \\
$\mu_{\eta \Delta}$ & $15 i \mathrm{GeV}$ & $7.1 i \mathrm{GeV}$ & $6 i \mathrm{GeV}$ \\
$m_{N_{1}}$ & $6 \mathrm{TeV}$ & $10 \mathrm{TeV}$ & $15 \mathrm{TeV}$ \\
$m_{N_{2}}$ & $6.6 \mathrm{TeV}$ & $11 \mathrm{TeV}$ & $16.5 \mathrm{TeV}$ \\
$m_{N_{3}}$ & $7.2 \mathrm{TeV}$ & $12 \mathrm{TeV}$ & $18 \mathrm{TeV}$ \\
$m_{\eta^{0}}$ & $600 \mathrm{GeV}$ & $1 \mathrm{TeV}$ & $1.5 \mathrm{TeV}$ \\
$\Delta m_{\eta^{0}}$ & $506 \mathrm{keV}$ & $300 \mathrm{keV}$ & $200 \mathrm{keV}$ \\
$m_{\eta^{ \pm}}$ & $606 \mathrm{GeV}$ & $1 \mathrm{TeV}$ & $1.5 \mathrm{TeV}$ \\
$m_{\Delta^{0}}$ & $1.2 \mathrm{TeV}$ & $2 \mathrm{TeV}$ & $3 \mathrm{TeV}$ \\
$m_{\Delta^{ \pm}}$ & $1.2 \mathrm{TeV}$ & $2 \mathrm{TeV}$ & $3 \mathrm{TeV}$ \\
$m_{\Delta^{ \pm \pm}}$ & $1.2 \mathrm{TeV}$ & $2 \mathrm{TeV}$ & $3 \mathrm{TeV}$ \\
$\lambda_{H}$ & 0.253 & 0.253 & 0.253 \\
$\lambda_{H \eta}$ & 0.19 & 0.56 & 0.91 \\
$\lambda_{H \eta}^{\prime}$ & -0.19 & -0.56 & -0.91 \\
$\lambda_{H \eta}^{\prime \prime}$ & $1 \times 10^{-5}$ & $1 \times 10^{-5}$ & $1 \times 10^{-5}$ \\
\hline \hline
\end{tabular}


coming from the standard decay of $N$ 's will not come into play as it requires nontrivial orthogonal matrix $\mathcal{O}$. The RHNs are taken to be much heavier than the $\eta$ particles to avoid the wash-out of lepton asymmetry from the inverse decay processes $L_{\alpha} \eta \rightarrow N_{i}$. For the BPs we take, the mass splitting $m_{\eta_{I}}-m_{\eta_{R}}$ (with $\eta_{I}$ the imaginary part from $\eta^{0}$ ) is larger than $100 \mathrm{keV}$ scale, such that the direct detection constraints for inelastic scattering of DM with nucleons [44-46] can be evaded.

The evolutions of the DM relic density $\Omega_{\mathrm{DM}} h^{2}$ and the baryon asymmetry $Y_{\Delta B}$ are evaluated using micrOMEGAs 5.0 [47] and the results are presented in Fig. 4. The time evolutions of DM relic density for BP1, BP2, and BP3 are denoted, respectively, by the red solid, dashed and dotdashed curves. For each choice of the DM mass, the maximal contribution to baryon asymmetry comes in the vicinity of the $s$-channel resonance in Fig. 3, i.e., when $2 m_{\eta} \rightarrow m_{\Delta}$. In Fig. 4 we have fixed the $\Delta$-mediator mass at the resonance point and have satisfied the required baryon asymmetry by fixing the trilinear coupling $\mu_{\eta \Delta}$ as shown in Table I.

The dependence of baryon asymmetry on the absolute value of the trilinear coupling $\left|\mu_{\eta \Delta}\right|$ and the triplet scalar mass $m_{\Delta}$ is shown in Fig. 5. For each of the three BPs given in Table I, we show the variation of $Y_{\Delta B}$ as function of the mediator mass for different values of $\mu_{\eta \Delta}$, as shown by the solid, dashed and dot-dashed lines with green, blue and red, which correspond respectively to BP1, BP2, and BP3. In the numerical calculations we have included the resonance

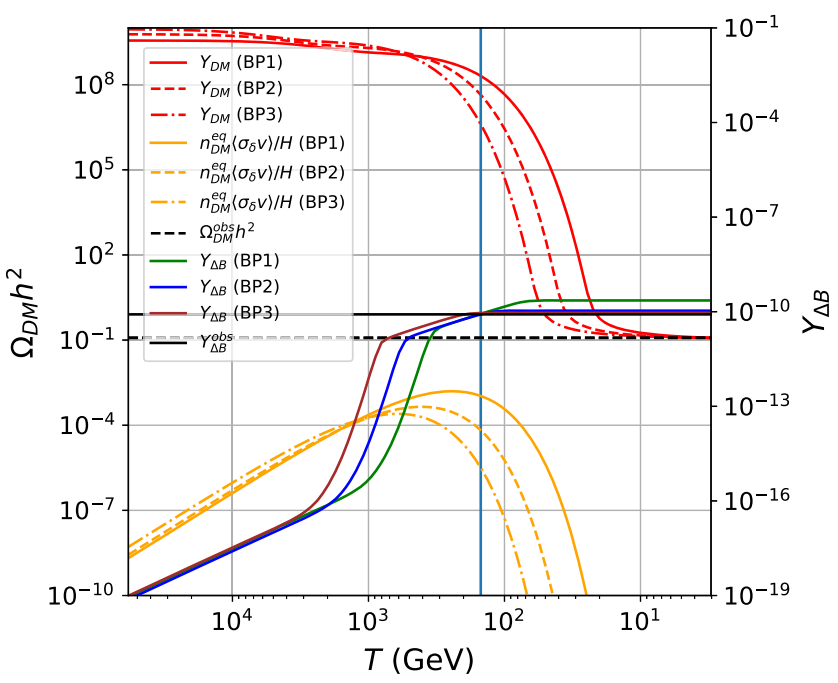

FIG. 4. Net baryon number density $Y_{\Delta B}$, DM density $Y_{\mathrm{DM}}$ and $n_{\mathrm{DM}}^{\mathrm{eq}}\langle\sigma v\rangle_{\delta} / H$ as functions of temperature $T$, for the three BPs in Table I. The solid horizontal black line indicates the observed baryon number density $Y_{\Delta B}^{\mathrm{obs}}=(8.718 \pm 0.004) \times 10^{-11}$, and the dashed horizontal black line indicates the central value of the observed DM relic density $\Omega_{\mathrm{DM}}^{\mathrm{obs}} h^{2}=0.120 \pm 0.001$ [1]. The vertical solid line represents the central value of the sphaleron freeze-out temperature $T_{\mathrm{sph}}=(131.7 \pm 2.3) \mathrm{GeV}$.

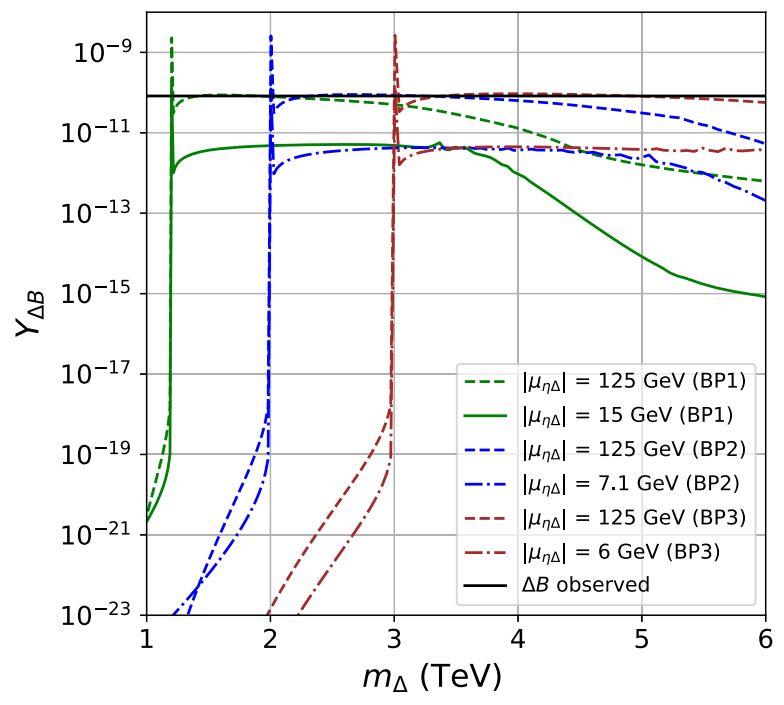

FIG. 5. Net baryon number density $Y_{\Delta B}$ as function of the $\Delta$ mediator mass, for three different values of $\left|\mu_{\eta \Delta}\right|$ (with the argument of $\pi / 2$ ) for each of the three BPs in Table I. The solid horizontal black line indicates the central value of the observed baryon number density $Y_{\Delta B}^{\mathrm{obs}}=(8.718 \pm 0.004) \times 10^{-11}$.

effect in Eq. (15) when the center-of-mass energy is close to the triplet scalar mass $m_{\Delta}$. The enhancement of baryon asymmetry at the resonance $m_{\Delta} \simeq 2 m_{\eta}$ can be clearly seen in Fig. 5. Note that in the narrow-width approximation, the thermal-averaged cross section $\langle\sigma v\rangle_{\delta}$ asymptotically reaches a finite value [cf. Eq. (C3)] which determines the height of the peak in Fig. 5, whereas the sharp drop right after the resonance is due to the Boltzmann suppression in Eq. (C3). As expected in Eq. (17), increasing the magnitude of $\mu_{\eta \Delta}$ results in a larger baryon asymmetry. We have fixed $\left|\mu_{\eta \Delta}\right|$ for each BP in Table I to be the minimum value for which the observed baryon asymmetry can be obtained at the resonance. Note that for larger trilinear couplings, one can also achieve the observed asymmetry away from the resonance point. This plateau region is due to a mutual cancellation between the $s$ - and $t$-channel contributions in Fig. 3, which in turn lowers the wash-out rate, and as a result, slightly enhances the baryon asymmetry.

The dependence of baryon asymmetry on the triplet VEV $v_{\Delta}$ can be seen in the plots in Fig. 6. Here the solid lines are for the evolution of $Y_{\Delta B}$ as function of $T$, and the dotted, dashed and dot-dashed lines denote the evolution of the thermally averaged cross sections $\langle\sigma v\rangle$, respectively for the processes $(\eta \eta \rightarrow L L)_{\delta}, \quad(\eta \eta \rightarrow L L)_{\text {tot }}$ and $(\eta \eta \rightarrow \mathrm{SMSM})$. The red, blue, green and magenta lines are respectively for the BPs with $\left|\mu_{\eta \Delta}\right|=1 \mathrm{GeV}, 10 \mathrm{GeV}$, $100 \mathrm{GeV}$ and $1 \mathrm{TeV}$, and the left and right panels are respectively for the VEVs of $v_{\Delta}=0.1 \mathrm{eV}$ and $v_{\Delta}=$ $100 \mathrm{eV}$. Other parameter are set to be the same as for $\mathrm{BP} 1$. When the VEV $v_{\Delta}$ gets larger, the Yukawa coupling $Y^{\Delta} \propto v_{\Delta}^{-1}$ will be smaller, so the wash-out effect will be suppressed and the resultant baryon asymmetry $Y_{\Delta B}$ will be 

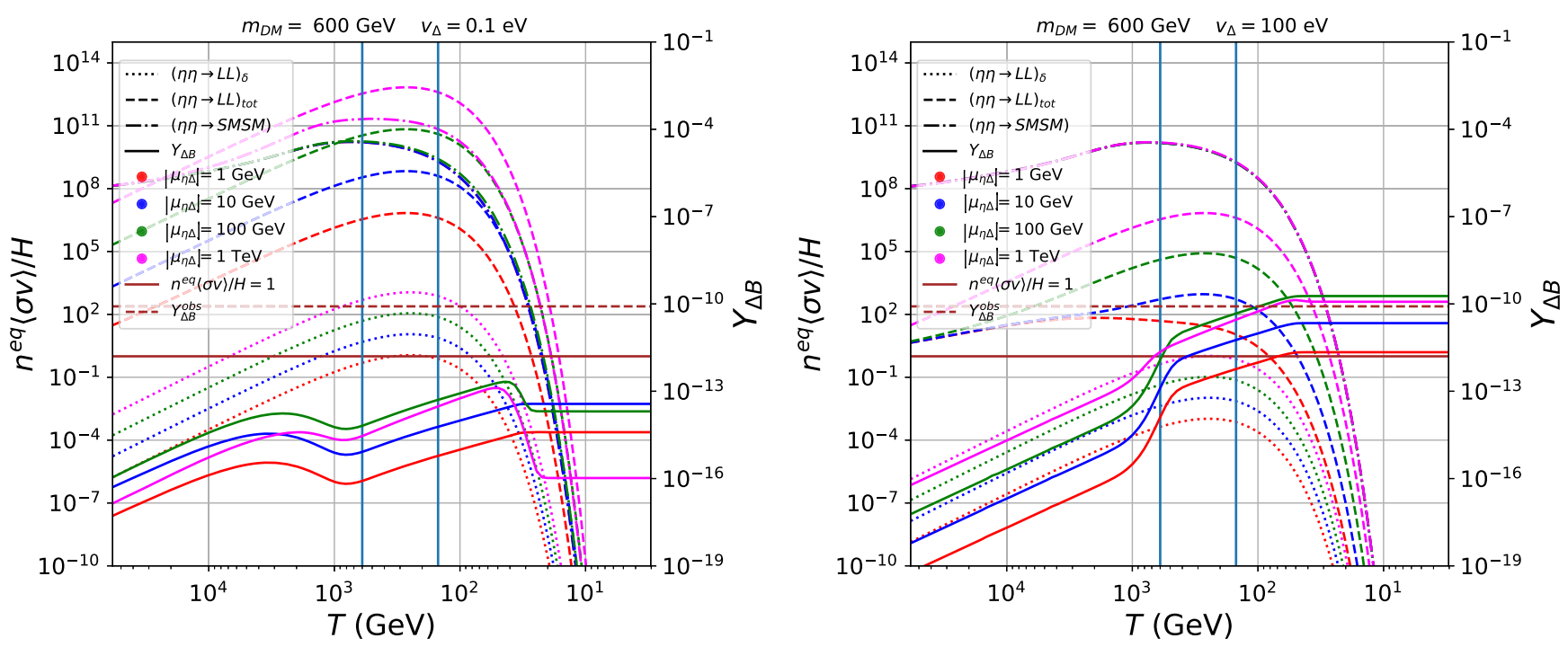

FIG. 6. Net baryon number density $Y_{\Delta B}$ and $n^{\text {eq }}\langle\sigma v\rangle / H$ for the processes $(\eta \eta \rightarrow L L)_{\delta},(\eta \eta \rightarrow L L)_{\text {tot }}$ and $(\eta \eta \rightarrow \mathrm{SMSM})$ as functions of temperature $T$, for the values of $\left|\mu_{\eta \Delta}\right|=1 \mathrm{GeV}, 10 \mathrm{GeV}, 100 \mathrm{GeV}$ and $1 \mathrm{TeV}$ for the BP1 in Table I, with $v_{\Delta}=0.1 \mathrm{eV}$ (left) and $v_{\Delta}=100 \mathrm{eV}$ (right). The solid horizontal black line indicates the central value of the observed baryon number density $Y_{\Delta B}^{\mathrm{obs}}=(8.718 \pm 0.004) \times 10^{-11}$, and the dashed horizontal black line indicates the central value of the observed DM relic density $\Omega_{\mathrm{DM}}^{\mathrm{obs}} h^{2}=0.120 \pm 0.001$. The vertical solid line represents the central value of the sphaleron freeze-out temperature $T_{\mathrm{sph}}=(131.7 \pm 2.3) \mathrm{GeV}$.

larger, as can be seen by comparing the left and right panels of Fig. 6.

Varying $v_{\Delta}$ and $\left|\mu_{\eta \Delta}\right|$ and fixing all other relevant parameters as in Table I, we obtain the allowed regions of $v_{\Delta}$ and $\left|\mu_{\eta \Delta}\right|$ which simultaneously satisfy the observed baryon number density $Y_{\Delta B}^{\mathrm{obs}}=(8.718 \pm 0.004) \times 10^{-11}$ and the observed DM relic density $\Omega_{\mathrm{DM}}^{\mathrm{obs}} h^{2}=0.120 \pm 0.001$, as shown by the shaded regions in Fig. 7 for the three BPs. ${ }^{4}$ From this parameter scan, we find both lower and upper bounds on the triplet VEV and the trilinear coupling for each BP:

\section{$\mathrm{BP} 1: 40 \mathrm{eV} \lesssim v_{\Delta} \lesssim 1.5 \mathrm{MeV}, \quad 0.3 \mathrm{GeV} \lesssim\left|\mu_{\eta \Delta}\right| \lesssim 80 \mathrm{GeV}$, \\ BP2: $20 \mathrm{eV} \lesssim v_{\Delta} \lesssim 1.2 \mathrm{MeV}, \quad 0.3 \mathrm{GeV} \lesssim\left|\mu_{\eta \Delta}\right| \lesssim 380 \mathrm{GeV}$, BP3: $10 \mathrm{eV} \lesssim v_{\Delta} \lesssim 20 \mathrm{MeV}, \quad 0.3 \mathrm{GeV} \lesssim\left|\mu_{\eta \Delta}\right| \lesssim 1.2 \mathrm{TeV}$.}

The lower limit on $\left|\mu_{\eta \Delta}\right|$ is set by the $Y_{\Delta B}$ requirement, while the upper limit is set by the relic density requirement, which is governed by the $\eta \eta \rightarrow W W$ process that is independent of $v_{\Delta}$ for most part of the parameter space. As for the limits on $v_{\Delta}$,

\footnotetext{
${ }^{4}$ To find the maximum allowed parameter space for $Y_{\Delta B}$, we take the maximal $C P$ phase for $\mu_{\eta \Delta}$ and keep all the points with $Y_{\Delta B} \geq Y_{\Delta B}^{\text {obs }}$. Since the $C P$-asymmetry depends on $\operatorname{Im}\left(\mu_{\eta \Delta}\right)$, cf. Eq. (15), we can always adjust the $C P$ phase accordingly for a fixed $\left|\mu_{\eta \Delta}\right|$ to get $Y_{\Delta B}=Y_{\Delta B}^{\text {obs }}$. Therefore, we only show $\left|\mu_{\eta \Delta}\right|$ and not its phase in Fig. 7.
}

when $v_{\Delta}$ is very small, the Yukawa coupling $Y^{\Delta} \propto v_{\Delta}^{-1}$ is so large that the washout effect from $L L \rightarrow \eta \eta$ is too strong. On the other hand, when $v_{\Delta}$ is very large the coupling $Y^{\Delta}$ is too small to produce sufficient baryon asymmetry. Although one would expect that the suppression of $Y^{\Delta}$ can be compensated

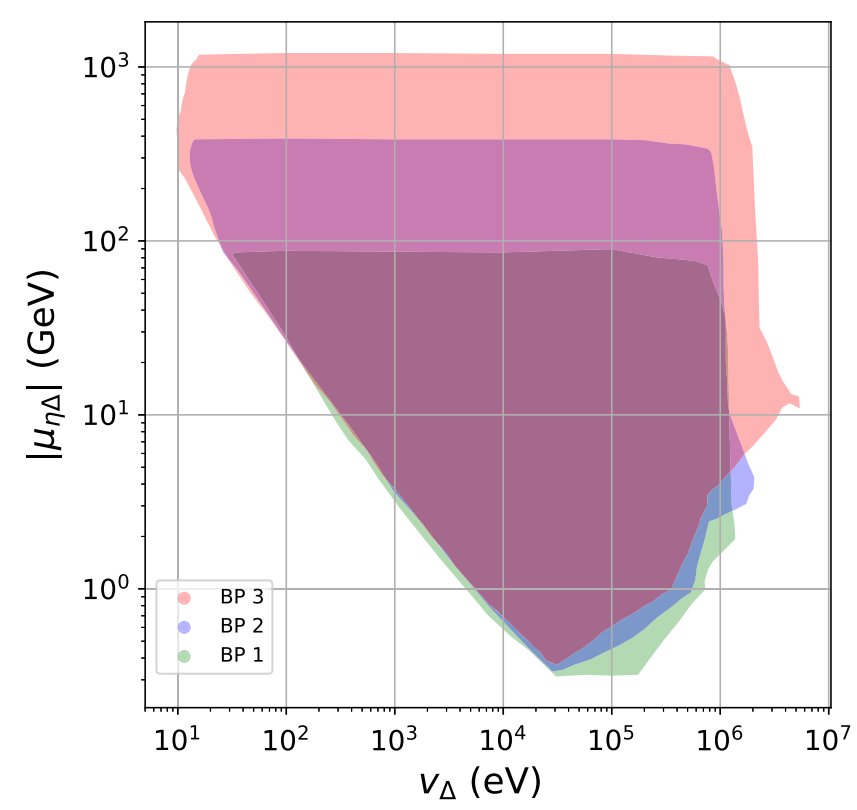

FIG. 7. Allowed parameter space of $v_{\Delta}$ and $\left|\mu_{\eta \Delta}\right|$ for the three BPs simultaneously satisfying the observed relic density and baryon asymmetry, with all other relevant parameters set to be the same as in Table I. 
by increasing $\mu_{\eta \Delta}$ [cf. Fig. 3 (left)], this in turn increases the mass splitting between $\eta_{R}$ and $\eta_{I}$, which decreases $Y^{N}$ [cf. Fig. 3 (right)] to maintain the neutrino mass. Due to this reason there is a sharp upper bound on $v_{\Delta}$.

The leptogenesis mechanism in this model can thus be directly tested at future lepton colliders by measuring the Yukawa couplings of doubly charged scalars [48], which is intimately related to $v_{\Delta}$ and the active neutrino masses and mixings via Eq. (13). This model can also be tested at future hadron and lepton colliders by searches of the beyond SM particles in the scotogenic and type-II seesaw models, as detailed in Sec. V.

\section{COLLIDER SIGNALS}

The BPs chosen for our model to simultaneously explain baryogenesis, DM and neutrino masses involve TeV-scale beyond SM scalars and heavy RHNs which can be directly tested at current and future high-energy colliders. For instance, the neutral and charged triplet scalars can be directly searched for at the Large Hadron Collider (LHC) $[49,50]$, as well as in future High-Luminosity LHC (HLLHC) [51-55], $100 \mathrm{TeV}$ hadron colliders [54,56] and lepton colliders [57], or indirectly probed in the highprecision low-energy experiments like MOLLER [58]. It is important to note here that the allowed range of $v_{\Delta}$ in Fig. 3 correspond to the Yukawa couplings $5 \times 10^{-9} \lesssim Y^{\Delta} \lesssim 3 \times$ $10^{-3}$ which give rise to prompt dilepton signals in the $\Delta^{ \pm \pm}$ decays for the triplet masses considered in Table I.

The charged $\eta^{ \pm}$scalars can be produced in association with the neutral DM particle $\eta^{0}$ through the $W$ boson, i.e., $p p \rightarrow W^{*} \rightarrow \eta^{ \pm} \eta^{0} \rightarrow \eta^{0} \eta^{0} W^{(*)}$ [59]. The inert doublet scalars can also be produced from their couplings to the SM $Z$ boson via $p p \rightarrow \eta_{R} \eta_{I} j$ or the SM Higgs through $p p \rightarrow \eta^{0} \eta^{0} j$ (with $j$ being an energetic jet) [60]. The inert doublet sector can then be constrained by the mono- $W$ $[61,62]$ and monojet $[63,64]$ searches at the LHC. Our model can in principle be distinguished from the pure scotogenic or pure type-II seesaw model at colliders using both inert doublet and triplet scalar signatures.

In the scotogenic model, the RHNs do not mix directly with the light active neutrinos. For our chosen BPs, the heavy RHNs are heavier than the inert doublet and can only be produced at high-energy colliders from the off-shell decay $\eta^{ \pm *} \rightarrow \ell_{\alpha}^{ \pm} N_{i}$, followed by $N_{i} \rightarrow \ell_{\alpha}^{ \pm} \eta^{\mp(*)} \rightarrow \ell_{\alpha}^{ \pm} \eta^{0} W^{\mp(*)}$. Due to the Majorana nature of the heavy RHNs, we can get same-sign dileptons, as in the Keung-Senjanović process [65], but now with significant missing transverse energy (MET) due to the presence of $\eta^{0}$ in the final state. The SM background for $\ell^{ \pm} \ell^{ \pm}+W+$ MET is expected to be higher than that without MET, and a detailed simulation is needed to estimate the prospects of RHN signals in this model at future colliders.

\section{CONCLUSION}

We have proposed a new technique to generate the observed baryon asymmetry of the universe only via the tree-level interference of $2 \rightarrow 2$ scatterings or $1 \rightarrow 3$ decays. The $C P$-violating asymmetry comes from the absorptive part of the propagators. We have illustrated this mechanism explicitly in a simple scotogenic model with type-II seesaw, in which the asymmetry is generated in the $\Delta L=2$ processes $\eta \eta \rightarrow L L$ mediated by $s$-channel triplet scalars and $t$ or $u$-channel RHNs. The neutrino masses receive contributions from both scotogenic and type-II seesaw mechanisms. The real part of the neutral component of the inert doublet $\eta$ serves as a DM candidate. As shown in Fig. 4 the baryon asymmetry and DM relic density are correlated and both can be matched to their observed values for (sub-) TeV inert doublet and triplet masses. The baryogenesis requirements impose both lower and upper bounds on the triplet VEV, as shown in Fig. 7, with testable consequences at future colliders.

\section{ACKNOWLEDGMENTS}

We thank Pei-Hong Gu for comments on the draft. A. D. thanks the Particle Theory Group at Washington University in St. Louis for warm hospitality, where this work was initiated. Y.Z. thanks Kaladi Babu for useful discussions, and the High Energy Theory Group at Oklahoma State University for warm hospitality, where this work was completed. The work of P. S. B. D. and Y. Z. was supported in part by the U.S. Department of Energy under Grant No. DE-SC0017987 and in part by the MCSS. This work was also supported in part by the Neutrino Theory Network Program under Grant No. DE-AC02-07CH11359. The work of S. K. K. and A. D. was supported by the NRF of Korea Grants No. 2017K1A3A7A09016430, and No. 2017R1A2B4006338.

\section{APPENDIX A: SCALAR POTENTIAL AND SCALAR MASSES}

The most general scalar potential for the SM Higgs doublet $H \equiv\left(H^{+}, H^{0}\right)$, inert doublet $\eta \equiv\left(\eta^{+}, \eta^{0}\right)$ and triplet $\Delta \equiv\left(\Delta^{++}, \Delta^{+}, \Delta^{0}\right)$ is given by

$$
\begin{aligned}
V= & -\mu_{H}^{2}\left(H^{\dagger} H\right)+\mu_{\eta}^{2}\left(\eta^{\dagger} \eta\right)-\mu_{\Delta}^{2} \operatorname{Tr}\left[\Delta^{\dagger} \Delta\right]+\left(\mu_{H \Delta} \tilde{H}^{\dagger} \Delta H+\mu_{\eta \Delta} \eta^{\dagger} \Delta^{\dagger} \tilde{\eta}+\text { H.c. }\right) \\
& +\lambda_{H}\left(H^{\dagger} H\right)^{2}+\lambda_{\eta}\left(\eta^{\dagger} \eta\right)^{2}+\lambda_{\Delta}\left\{\operatorname{Tr}\left[\Delta^{\dagger} \Delta\right]\right\}^{2}+\lambda_{\Delta}^{\prime} \operatorname{Tr}\left[\Delta^{\dagger} \Delta \Delta^{\dagger} \Delta\right]+\lambda_{H \eta}\left|H^{\dagger} \eta\right|^{2}+\lambda_{H \eta}^{\prime}\left(H^{\dagger} H\right)\left(\eta^{\dagger} \eta\right) \\
& +\lambda_{H \eta}^{\prime \prime}\left(\left(H^{\dagger} \eta\right)^{2}+\text { H.c. }\right)+\lambda_{H \Delta}\left(H^{\dagger} H\right) \operatorname{Tr}\left[\Delta^{\dagger} \Delta\right]+\lambda_{H \Delta}^{\prime} \operatorname{Tr}\left[H^{\dagger} \Delta \Delta^{\dagger} H\right]+\lambda_{\eta \Delta}\left(\eta^{\dagger} \eta\right) \operatorname{Tr}\left[\Delta^{\dagger} \Delta\right]+\lambda_{\eta \Delta}^{\prime} \operatorname{Tr}\left[\eta^{\dagger} \Delta \Delta^{\dagger} \eta\right],
\end{aligned}
$$


where $\tilde{H}=i \sigma_{2} H^{*}, \tilde{\eta}=i \sigma_{2} \eta^{*}$ and the mass parameters $\mu_{H, \eta, \Delta}^{2}>0$ so that both $H$ and $\Delta$ obtain nonvanishing VEVs, i.e., $\left\langle H^{0}\right\rangle=v \simeq 246 \mathrm{GeV}$ and $\left\langle\Delta^{0}\right\rangle=v_{\Delta}$. To generate the baryon asymmetry from the tree-level interference effect, the coupling $\mu_{\eta \Delta}$ is assumed to be complex, and all other parameters in Eq. (A1) are assumed to be real.

The physical masses for the neutral and charged scalars can be obtained from minimization of the scalar potential in Eq. (A1). Note that here the doublet $\eta$ is odd under the $Z_{2}$ symmetry, which is essential to provide a DM candidate, and does not mix the SM Higgs and the triplet. In particular, when we take the first-order derivative of the potential with respect to the VEVs $v$ and $v_{\Delta}$, the solutions of the tadpole equations for $\left\{\mu_{H}^{2}, \mu_{\Delta}^{2}\right\}$ are given by

$$
\begin{aligned}
& \mu_{H}^{2}=\frac{1}{2}\left(\lambda_{H} v^{2}-2 \sqrt{2} \mu_{H \Delta} v_{\Delta}+\lambda_{H \Delta} v_{\Delta}^{2}\right), \\
& \mu_{\Delta}^{2}=\frac{1}{2}\left(\lambda_{\Delta} v_{\Delta}^{2}-2 \sqrt{2} \frac{\mu_{H \Delta}}{v_{\Delta}} v^{2}+\lambda_{H \Delta} v^{2}\right) .
\end{aligned}
$$

After replacing $\left\{\mu_{H}^{2}, \mu_{\Delta}^{2}\right\}$ in the scalar potential, the mass matrix for the real scalars reads

$$
\mathcal{M}^{0}=\left(\begin{array}{cc}
\lambda_{H} v^{2} & -\sqrt{2} \mu_{H \Delta} v \\
-\sqrt{2} \mu_{H \Delta} v & \frac{\mu_{H \Delta} v^{2}}{\sqrt{2} v_{\Delta}}
\end{array}\right),
$$

from which we can get two mass eigenvalues for the real component from $H^{0}$ and the scalar $\Delta_{R}^{0}$ which is the real part of $\Delta^{0}$. In the case of $\mu_{H \Delta} \sim \mathcal{O}(100) \mathrm{keV}$, the two $C P$-even scalar masses turn out to be

$$
m_{h}^{2} \simeq \lambda_{H} v^{2}, \quad m_{\Delta_{R}^{0}}^{2} \simeq \frac{\mu_{H \Delta} v^{2}}{\sqrt{2} v_{\Delta}}
$$

with the first one $(h)$ identified as the SM-like Higgs boson. The masses of the pseudoscalar and the charged scalars from the triplet are respectively

$$
\begin{gathered}
m_{\Delta_{I}}^{2}=\frac{\mu_{H \Delta}}{\sqrt{2} v_{\Delta}}\left(v^{2}+4 v_{\Delta}^{2}\right), \\
m_{\Delta^{ \pm}}^{2}=m_{\Delta^{ \pm \pm}}^{2}=\left(\frac{\mu_{H \Delta}}{\sqrt{2} v_{\Delta}}+\frac{1}{4} \lambda_{H \Delta}^{\prime}\right)\left(v^{2}+2 v_{\Delta}^{2}\right) .
\end{gathered}
$$

Finally the masses for real scalar $\eta_{R}$, pseudoscalar $\eta_{I}$ and the charged scalars $\eta^{ \pm}$from the $Z_{2}$-odd doublet $\eta$ are respectively

$$
\begin{aligned}
m_{\eta_{R, I}}^{2}= & \frac{1}{2}\left[2 \mu_{\eta}^{2}+\left(\lambda_{H \eta}+\lambda_{H \eta}^{\prime} \pm \lambda_{H \eta}^{\prime \prime}\right) v^{2}\right. \\
& \left.+\left(\lambda_{\eta \Delta} v_{\Delta} \mp 2 \sqrt{2}\left|\mu_{\eta \Delta}\right|\right) v_{\Delta}\right],
\end{aligned}
$$

$$
m_{\eta^{ \pm}}^{2}=\frac{1}{2}\left(2 \mu_{\eta}^{2}+\lambda_{H \eta} v^{2}+\left(\lambda_{\eta \Delta}+\lambda_{\eta \Delta}^{\prime}\right) v_{\Delta}^{2}\right)
$$

\section{APPENDIX B: THERMAL CROSS SECTIONS}

The general expression for the thermally averaged cross section for the processes in Eqs. (18) and (19) is [47]

$$
\begin{aligned}
\langle\sigma v\rangle\left(i_{1} i_{2} \rightarrow f_{1} f_{2}\right)= & \frac{1}{2 T m_{i_{1}}^{2} K_{2}\left(m_{i_{1}} / T\right) m_{i_{2}}^{2} K_{2}\left(m_{i_{2}} / T\right)} \\
& \times \int_{s_{\text {in }}}^{\infty} \int_{-1}^{1} \frac{1}{32 \pi} \frac{|\mathcal{M}|^{2}}{\sqrt{s}} p_{i_{1} i_{2}} p_{f_{1} f_{2}} \\
& \times K_{1}(\sqrt{s} / T) \mathrm{d} s \mathrm{~d} \cos \theta
\end{aligned}
$$

where $T$ is the temperature, $K_{i}$ the modified Bessel functions of order $i, \mathcal{M}$ is the amplitude for the process $i_{1} i_{2} \rightarrow f_{1} f_{2}$, and

$$
\begin{gathered}
p_{i j} \equiv \frac{1}{2} \sqrt{\lambda\left(s, m_{i}^{2}, m_{j}^{2}\right) / s}, \\
s_{\text {in }} \equiv \max \left[\left(m_{i_{1}}+m_{i_{2}}\right)^{2},\left(m_{f_{1}}+m_{f_{2}}\right)^{2}\right], \\
\lambda(x, y, z) \equiv x^{2}+y^{2}+z^{2}+2 x y+2 x z+2 y z .
\end{gathered}
$$

In Eq. (19), $\langle\sigma v\rangle_{\text {tot }}(\eta \eta \rightarrow L L)$ and $\langle\sigma v\rangle(\eta \bar{L} \rightarrow \eta L)$ are respectively for the amplitudes:

$$
\begin{aligned}
\left|\mathcal{M}_{\mathrm{tot}}(\eta \eta \rightarrow L L)\right|^{2}= & \frac{\hat{m}_{\nu}^{2}}{v_{\Delta}^{2}} \frac{F_{\mathrm{I}}^{2}\left|\mu_{\eta \Delta}\right|^{2} s}{\left(s-m_{\Delta}^{2}\right)^{2}+m_{\Delta}^{2} \Gamma_{\Delta}^{2}} \\
& +\sum_{i} F_{\mathrm{II}}^{2} \frac{\hat{m}_{\nu}^{2}}{\Lambda_{i i}^{2}} m_{N_{i}}^{2} s\left[\frac{1}{t-m_{N_{i}}^{2}}+\frac{1}{u-m_{N_{i}}^{2}}\right]^{2} \\
& +\frac{F_{\mathrm{I}} F_{\mathrm{II}}\left|\mu_{\eta \Delta}\right|\left(s-m_{\Delta}^{2}\right)}{\left(s-m_{\Delta}^{2}\right)^{2}+m_{\Delta}^{2} \Gamma_{\Delta}^{2}} \sum_{i} \frac{\hat{m}_{\nu}^{2}}{\Lambda_{i i} v_{\Delta}} m_{N_{i}} s \\
& \times\left[\frac{1}{t-m_{N_{i}}^{2}}+\frac{1}{u-m_{N_{i}}^{2}}\right], \\
|\mathcal{M}(\eta \bar{L} \rightarrow \eta L)|^{2}= & \frac{\hat{m}_{\nu}^{2}}{v_{\Delta}^{2}} \frac{F_{\mathrm{I}}^{2}\left|\mu_{\eta \Delta}\right|^{2}\left(m_{\eta}^{2}-t\right)}{\left(t-m_{\Delta}^{2}\right)^{2}} \\
& +\sum_{i} \frac{\hat{m}_{\nu}^{2}}{\Lambda_{i i}^{2}} \frac{F_{\mathrm{II}}^{2} m_{N_{i}}^{2} s}{\left(s-m_{N_{i}}^{2}\right)^{2}+m_{N_{i}}^{2} \Gamma_{N_{i}}^{2}} \\
& +\frac{F_{\mathrm{I}} F_{\mathrm{II}}\left|\mu_{\eta \Delta}\right|\left(m_{\eta}^{2}-t\right)}{\left(t-m_{\Delta}^{2}\right)^{2}} \sum_{i} \frac{\hat{m}_{\nu}^{2}}{\Lambda_{i i} v_{\Delta}} m_{N_{i}} s \\
& \times\left[\frac{s-m_{N_{i}}^{2}}{\left(s-m_{N_{i}}^{2}\right)^{2}+m_{N_{i}}^{2} \Gamma_{N_{i}}^{2}}\right] .
\end{aligned}
$$

The cross section $\langle\sigma v\rangle(\eta \eta \rightarrow$ SMSM) in Eq. (18) can be found in $[37,38]$, with "SM SM" referring to the all the 
possible channels involving the quarks, leptons, scalar and gauge bosons in the SM.

\section{APPENDIX C: MAXIMUM ASYMMETRY}

In Eq. (19) $\langle\sigma v\rangle_{\delta}(\eta \eta \rightarrow L L)$ is for the amplitude given in Eq. (15) which produces the lepton asymmetry. In the narrow-width approximation, the asymmetry in Eq. (15) at the resonance point simplifies to

$$
\begin{aligned}
\delta \simeq & 4 \sum_{i} \operatorname{Im}\left[\mu_{\eta \Delta}\left\{Y^{N} Y^{\Delta^{*}}\left(Y^{N}\right)^{\top}\right\}_{i i}\right] \\
& \times s m_{N_{i}} \delta\left(s-m_{\Delta}^{2}\right)\left[\frac{1}{t-m_{N_{i}}^{2}}+\frac{1}{u-m_{N_{i}}^{2}}\right],
\end{aligned}
$$

where we have used

$$
\frac{m_{\Delta} \Gamma_{\Delta}}{\left(s-m_{\Delta}^{2}\right)^{2}+m_{\Delta}^{2} \Gamma_{\Delta}^{2}} \stackrel{\Gamma_{\Delta} / m_{\Delta} \rightarrow 0}{\longrightarrow} \pi \delta\left(s-m_{\Delta}^{2}\right) .
$$

Plugging the amplitude (C1) back into Eq. (B1) and integrating over $s$, we get

$$
\begin{aligned}
&\langle\sigma v\rangle_{\delta}(\eta \eta \rightarrow L L) \\
&=\frac{z}{128 \pi^{2} m_{\eta}^{5} K_{2}^{2}(z)} \int_{s_{\mathrm{in}}}^{\infty} d s \frac{p_{\eta \eta} p_{L L}}{\sqrt{s}} K_{1}\left(\frac{\sqrt{s} z}{m_{\eta}}\right) \delta \\
& \simeq \frac{4 \pi}{m_{\eta}^{2}} \frac{r_{\Delta}^{4}}{\tilde{\mu}_{\eta \Delta}^{2} \sum_{\alpha, \beta}\left|Y_{\alpha \beta}\right|^{2}} \Gamma_{\Delta \rightarrow \eta \eta} \Gamma_{\Delta \rightarrow L L} \\
& \times \sum_{i} \operatorname{Im}\left[\mu_{\eta \Delta}\left\{Y^{N} Y^{\Delta^{*}}\left(Y^{N}\right)^{\top}\right\}_{i i}\right] \frac{K_{1}\left(r_{\Delta} z\right)}{r_{N_{i}} K_{2}^{2}(z)},
\end{aligned}
$$

where $\tilde{\mu}_{\eta \Delta}=\mu_{\eta \Delta} / m_{\Delta}, r_{i}=m_{i} / m_{\eta}, p_{i j}$ and $s_{\text {in }}$ are defined in Eqs. (B2) and (B3) respectively, and

$$
\begin{gathered}
\Gamma_{\Delta \rightarrow \eta \eta}=\frac{1}{16 \pi} \tilde{\mu}_{\eta \Delta}^{2} p_{\eta \eta}, \\
\Gamma_{\Delta \rightarrow L L}=\frac{1}{16 \pi}\left|Y_{\alpha \beta}\right|^{2} p_{L L} .
\end{gathered}
$$

It can be seen from Eq. (C3) that the thermally averaged asymmetry asymptotically reaches a finite value at the resonance, which determines the height of the peak in Fig. 5.
[1] N. Aghanim et al. (Planck Collaboration), Planck 2018 results. VI. Cosmological parameters, arXiv:1807.06209.

[2] A. D. Sakharov, Violation of CP invariance, C asymmetry, and baryon asymmetry of the universe, Pis'ma Zh. Eksp. Teor. Fiz. 5, 32 (1967).

[3] S. Dimopoulos and L. Susskind, Baryon asymmetry in the very early universe, Phys. Lett. 81B, 416 (1979).

[4] S. Weinberg, Cosmological Production of Baryons, Phys. Rev. Lett. 42, 850 (1979).

[5] M. Fukugita and T. Yanagida, Baryogenesis without grand unification, Phys. Lett. B 174, 45 (1986).

[6] J. M. Cline, Baryogenesis, in Les Houches Summer School -Session 86: Particle Physics and Cosmology: The Fabric of Spacetime Les Houches, France, 2006 (2006).

[7] S. Davidson, E. Nardi, and Y. Nir, Leptogenesis, Phys. Rep. 466, 105 (2008).

[8] D. V. Nanopoulos and S. Weinberg, Mechanisms for cosmological baryon production, Phys. Rev. D 20, 2484 (1979).

[9] R. Adhikari and R. Rangarajan, Baryon number violation in particle decays, Phys. Rev. D 65, 083504 (2002).

[10] A. Bhattacharya, R. Gandhi, and S. Mukhopadhyay, Revisiting the implications of $C P T$ and unitarity for baryogenesis and leptogenesis, Phys. Rev. D 89, 116014 (2014).

[11] M. Yoshimura, Unified Gauge Theories and the Baryon Number of the Universe, Phys. Rev. Lett. 41, 281 (1978); Erratum, Phys. Rev. Lett.42, 746 (1979).

[12] L. Bento and Z. Berezhiani, Leptogenesis Via Collisions: The Lepton Number Leaking to the Hidden Sector, Phys. Rev. Lett. 87, 231304 (2001).
[13] I. Baldes, N. F. Bell, K. Petraki, and R. R. Volkas, ParticleAntiparticle Asymmetries from Annihilations, Phys. Rev. Lett. 113, 181601 (2014).

[14] I. Baldes, N. F. Bell, A. Millar, K. Petraki, and R. R. Volkas, The role of $C P$ violating scatterings in baryogenesis-case study of the neutron portal, J. Cosmol. Astropart. Phys. 11 (2014) 041.

[15] G. R. Farrar and G. Zaharijas, Dark Matter and the Baryon Asymmetry, Phys. Rev. Lett. 96, 041302 (2006).

[16] P.-H. Gu and U. Sarkar, Annihilating leptogenesis, Phys. Lett. B 679, 118 (2009).

[17] Y. Cui, L. Randall, and B. Shuve, A WIMPy baryogenesis miracle, J. High Energy Phys. 04 (2012) 075.

[18] J. Kumar and P. Stengel, WIMPy leptogenesis with absorptive final state interactions, Phys. Rev. D 89, 055016 (2014).

[19] A. Dasgupta, C. Hati, S. Patra, and U. Sarkar, A minimal model of TeV scale WIMPy leptogenesis, arXiv: 1605.01292.

[20] D. Borah, A. Dasgupta, and S. K. Kang, Leptogenesis from dark matter annihilations in scotogenic model, Eur. Phys. J. C 80, 498 (2020).

[21] E. Ma, Verifiable radiative seesaw mechanism of neutrino mass and dark matter, Phys. Rev. D 73, 077301 (2006).

[22] M. Magg and C. Wetterich, Neutrino mass problem and gauge hierarchy, Phys. Lett. 94B, 61 (1980).

[23] J. Schechter and J. W. F. Valle, Neutrino masses in $\mathrm{SU}(2) \times \mathrm{U}(1)$ theories, Phys. Rev. D 22, 2227 (1980).

[24] T. P. Cheng and L.-F. Li, Neutrino masses, mixings and oscillations in $\mathrm{SU}(2) \times \mathrm{U}(1)$ models of electroweak interactions, Phys. Rev. D 22, 2860 (1980). 
[25] R. N. Mohapatra and G. Senjanović, Neutrino masses and mixings in gauge models with spontaneous parity violation, Phys. Rev. D 23, 165 (1981).

[26] G. Lazarides, Q. Shafi, and C. Wetterich, Proton lifetime and fermion masses in an SO(10) model, Nucl. Phys. B181, 287 (1981).

[27] P. S. B. Dev, P. Di Bari, B. Garbrecht, S. Lavignac, P. Millington, and D. Teresi, Flavor effects in leptogenesis, Int. J. Mod. Phys. A 33, 1842001 (2018).

[28] M. Drewes, B. Garbrecht, P. Hernandez, M. Kekic, J. Lopez-Pavon, J. Racker, N. Rius, J. Salvado, and D. Teresi, ARS Leptogenesis, Int. J. Mod. Phys. A 33, 1842002 (2018).

[29] A. Sirlin, Theoretical Considerations Concerning the Z0 Mass, Phys. Rev. Lett. 67, 2127 (1991).

[30] P. A. Grassi, B. A. Kniehl, and A. Sirlin, Width and Partial Widths of Unstable Particles, Phys. Rev. Lett. 86, 389 (2001).

[31] A. Pilaftsis and T. E. J. Underwood, Resonant leptogenesis, Nucl. Phys. B692, 303 (2004).

[32] P. S. B. Dev, M. Garny, J. Klaric, P. Millington, and D. Teresi, Resonant enhancement in leptogenesis, Int. J. Mod. Phys. A 33, 1842003 (2018).

[33] S. Choubey and A. Kumar, Inflation and dark matter in the inert doublet model, J. High Energy Phys. 11 (2017) 080.

[34] D. Borah, P. S. B. Dev, and A. Kumar, TeV scale leptogenesis, inflaton dark matter and neutrino mass in a scotogenic model, Phys. Rev. D 99, 055012 (2019).

[35] A. Merle and M. Platscher, Running of radiative neutrino masses: The scotogenic model revisited, J. High Energy Phys. 11 (2015) 148.

[36] J. A. Casas and A. Ibarra, Oscillating neutrinos and $\mu \rightarrow e \gamma$, Nucl. Phys. B618, 171 (2001).

[37] R. Barbieri, L. J. Hall, and V.S. Rychkov, Improved naturalness with a heavy Higgs: An alternative road to LHC physics, Phys. Rev. D 74, 015007 (2006).

[38] L. Lopez Honorez, E. Nezri, J. F. Oliver, and M. H. G. Tytgat, The inert doublet model: An archetype for dark matter, J. Cosmol. Astropart. Phys. 02 (2007) 028.

[39] J. A. Harvey and M. S. Turner, Cosmological baryon and lepton number in the presence of electroweak fermion number violation, Phys. Rev. D 42, 3344 (1990).

[40] V. A. Kuzmin, V. A. Rubakov, and M. E. Shaposhnikov, On the anomalous electroweak baryon number nonconservation in the early universe, Phys. Lett. 155B, 36 (1985).

[41] M. D'Onofrio, K. Rummukainen, and A. Tranberg, Sphaleron Rate in the Minimal Standard Model, Phys. Rev. Lett. 113, 141602 (2014).

[42] F. Staub, SARAH 4: A tool for (not only SUSY) model builders, Comput. Phys. Commun. 185, 1773 (2014).

[43] W. Porod and F. Staub, SPheno 3.1: Extensions including flavour, $C P$-phases and models beyond the MSSM, Comput. Phys. Commun. 183, 2458 (2012).

[44] X. Chen et al. (PandaX-II Collaboration), Exploring the dark matter inelastic frontier with 79.6 days of PandaX-II data, Phys. Rev. D 96, 102007 (2017).

[45] E. Aprile et al. (XENON Collaboration), Search for WIMP inelastic scattering off xenon nuclei with XENON100, Phys. Rev. D 96, 022008 (2017).
[46] T. Suzuki et al. (XMASS Collaboration), Search for WIMP${ }^{129} \mathrm{Xe}$ inelastic scattering with particle identification in XMASS-I, Astropart. Phys. 110, 1 (2019).

[47] G. Bélanger, F. Boudjema, A. Goudelis, A. Pukhov, and B. Zaldivar, micrOMEGAs5.0: Freeze-in, Comput. Phys. Commun. 231, 173 (2018).

[48] P. S. B. Dev, R. N. Mohapatra, and Y. Zhang, Probing TeV scale origin of neutrino mass at future lepton colliders via neutral and doubly-charged scalars, Phys. Rev. D 98, 075028 (2018).

[49] M. Aaboud et al. (ATLAS Collaboration), Search for doubly charged Higgs boson production in multi-lepton final states with the ATLAS detector using protonproton collisions at $\sqrt{s}=13 \mathrm{TeV}$, Eur. Phys. J. C 78, 199 (2018).

[50] CMS Collaboration, A search for doubly-charged Higgs boson production in three and four lepton final states at $\sqrt{s}=13 \mathrm{TeV}$, Technical Report No. CMS-PAS-HIG16-036, CERN, Geneva, 2017. http://cds.cern.ch/record/ 2242956.

[51] P. Fileviez Perez, T. Han, G.-y. Huang, T. Li, and K. Wang, Neutrino masses and the CERN LHC: Testing type II seesaw, Phys. Rev. D 78, 015018 (2008).

[52] A. Melfo, M. Nemevsek, F. Nesti, G. Senjanović, and Y. Zhang, Type II Seesaw at LHC: The roadmap, Phys. Rev. D 85, 055018 (2012).

[53] M. Mitra, S. Niyogi, and M. Spannowsky, Type-II Seesaw model and multilepton signatures at hadron colliders, Phys. Rev. D 95, 035042 (2017).

[54] P. S. B. Dev and Y. Zhang, Displaced vertex signatures of doubly charged scalars in the type-II seesaw and its left-right extensions, J. High Energy Phys. 10 (2018) 199.

[55] S. Antusch, O. Fischer, A. Hammad, and C. Scherb, Low scale type II seesaw: Present constraints and prospects for displaced vertex searches, J. High Energy Phys. 02 (2019) 157.

[56] Y. Du, A. Dunbrack, M. J. Ramsey-Musolf, and J.-H. Yu, Type-II seesaw scalar triplet model at a $100 \mathrm{TeV} p p$ collider: Discovery and Higgs portal coupling determination, J. High Energy Phys. 01 (2019) 101.

[57] P. Agrawal, M. Mitra, S. Niyogi, S. Shil, and M. Spannowsky, Probing the Type-II seesaw mechanism through the production of Higgs bosons at a lepton collider, Phys. Rev. D 98, 015024 (2018).

[58] P. S. B. Dev, M. J. Ramsey-Musolf, and Y. Zhang, Doublycharged scalars in the Type-II seesaw mechanism: Fundamental symmetry tests and high-energy searches, Phys. Rev. D 98, 055013 (2018).

[59] N. Wan, N. Li, B. Zhang, H. Yang, M.-F. Zhao, M. Song, G. Li, and J.-Y. Guo, Searches for dark matter via mono-W production in inert doublet model at the LHC, Commun. Theor. Phys. 69, 617 (2018).

[60] I. M. vila, V. De Romeri, L. Duarte, and J. W. F. Valle, Minimalistic scotogenic scalar dark matter, arXiv: 1910.08422 .

[61] A. M. Sirunyan et al. (CMS Collaboration), Search for new physics in final states with an energetic jet or a hadronically decaying $W$ or $Z$ boson and transverse momentum imbalance at $\sqrt{s}=13 \mathrm{TeV}$, Phys. Rev. D 97, 092005 (2018). 
[62] M. Aaboud et al. (ATLAS Collaboration), Search for dark matter in events with a hadronically decaying vector boson and missing transverse momentum in $p p$ collisions at $\sqrt{s}=$ $13 \mathrm{TeV}$ with the ATLAS detector, J. High Energy Phys. 10 (2018) 180.

[63] V. Khachatryan et al. (CMS Collaboration), Search for dark matter, extra dimensions, and unparticles in monojet events in proton-proton collisions at $\sqrt{s}=8 \mathrm{TeV}$, Eur. Phys. J. C 75, 235 (2015).
[64] M. Aaboud et al. (ATLAS Collaboration), Search for dark matter and other new phenomena in events with an energetic jet and large missing transverse momentum using the ATLAS detector, J. High Energy Phys. 01 (2018) 126.

[65] W.-Y. Keung and G. Senjanović, Majorana Neutrinos and the Production of the Right-handed Charged Gauge Boson, Phys. Rev. Lett. 50, 1427 (1983). 